\title{
Microphase segregation in the melts of bottlebrush block copolymers.
}

\author{
Ekaterina B. Zhulina ${ }^{1}$, Sergei S.Sheiko ${ }^{1,2}$, \\ Andrey V.Dobrynin ${ }^{3}$, Oleg V.Borisov ${ }^{1,4}$ \\ ${ }^{1}$ Institute of Macromolecular Compounds
}

of the Russian Academy of Sciences, St.Petersburg, Russia

${ }^{2}$ Department of Chemistry University of North Carolina at Chapel Hill, 27599, USA

${ }^{3}$ Department of Polymer Science, University of Akron, Akron, Ohio 44325, USA

${ }^{4}$ Institut des Sciences Analytiques et de Physico-Chimie pour l'Environnement et les Matériaux, UMR 5254 CNRS UPPA, Pau, France

March 17, 2020

Supporting Information

\section{Theoretical model}

Our calculation of the conformational entropy of the blocks tethered to $A / B$ interface is based on the SS-SCF approximation according to which the molecular potentials $U_{A}$ and $U_{B}$ acting on monomers in $A$-domains and $B$-matrixes of microphase segregated melt are parabolic,

$$
\frac{U_{A}(z)}{k_{B} T}=\frac{3}{2 l_{A} b_{A}} \kappa_{A}^{2}\left(R_{i}^{2}-z^{2}\right) ; \quad \frac{U_{B}(z)}{k_{B} T}=\frac{3}{2 l_{B} b_{B}} \kappa_{B}^{2}\left(D_{i}^{2}-z^{2}\right)
$$

Here $R_{i}$ is radius of $A$-domain, $D_{i}+R_{i}$ is half-distance between centers of neighboring $A$-domains in morphology $i$, and topological coefficients $\kappa_{A}$ and $\kappa_{B}$ of the blocks are treated as known parameters. 


\subsection{Free energy of domain block $A$}

The elastic free energy of block $A$ in the domain with radius $R_{i}$ can be presented as

$$
F_{A}^{(i)}=\int_{0}^{R_{i}} f_{\text {elastic }}^{(A, i)}(z) s(z) d z
$$

The density of the elastic free energy $f_{\text {elastic }}^{(A, i)}$ is given by

$$
\frac{f_{\text {elastic }}^{(A, i)}(z)}{k_{B} T}=\frac{3 \kappa_{A}^{2}}{2 s(z) v_{A} l_{A} b_{A}} \int_{z}^{R_{i}} z^{\prime} \phi_{A}\left(z^{\prime}\right) s\left(z^{\prime}\right) d z^{\prime}, \quad 0 \leq z \leq R
$$

with area per molecule $s(z)$ decreasing as a function of distance $z$ from the $A / B$ interface as

$$
s(z)=s \cdot\left(\frac{R-z}{R}\right)^{i-1}=\frac{i N_{A} v_{A}^{3}}{R_{i}} \cdot\left(\frac{R-z}{R}\right)^{i-1} \quad i=1,2,3
$$

Taking into account uniform polymer density in the $A$-domain, $\phi_{A}(z)=$ const $\approx 1$, and performing integration in eq 2 one finds

$$
\frac{F_{A}^{(i)}}{k_{B} T}=\frac{\mathrm{b}_{i} R_{i}^{2}}{N_{A} l_{A} b_{A}} \eta_{A}^{2}
$$

where

$$
\mathrm{b}_{i}= \begin{cases}\pi^{2} / 8, & i=1 \\ \pi^{2} / 16, & i=2 \\ 3 \pi^{2} / 80, & i=3\end{cases}
$$

are morphology-dependent numerical prefactors that coincide with whose obtained earlier $^{2}$ for the case of linear core-forming block (corresponding to $\eta_{A}=1$ ). As it was proposed in $\mathrm{ref}^{3}$ and follows from eq 5 , prefactor $\eta_{A}^{2}$ determines the increase in elastic free energy of the branched block $A$ compared to linear block with the same number $N_{A}$ of monomer units stretched to the same extent.

\subsection{Free energy of matrix block $B$}

The conformational free energy of blocks $B$ tethered to the surface of the $A$-domains can be expressed as

$$
F_{B}^{(i)}=\int_{0}^{D} f_{\text {elastic }}^{(B, i)}(z) s(z) d z
$$


where $f_{\text {elastic }}^{(B, i)}(z)$ is density of the elastic free energy. As long as blocks $B$ exhibit the Gaussian elasticity on all length scales, $f_{\text {elastic }}^{(B, i)}(z)$ can be expressed as

$$
\frac{f_{\text {elastic }}^{(B, i)}(z)}{k_{B} T}=\frac{T^{(B, i)}(z)}{2 k_{B} T}=\frac{3 \kappa_{B}^{2}}{2 s(z) v_{B} l_{B} b_{B}} \int_{z}^{D} z^{\prime} \phi_{B}\left(z^{\prime}\right) s\left(z^{\prime}\right) d z^{\prime}
$$

where $T^{(B, i)}(z)$ is the flux of elastic tension per unit area, $\phi_{B}(z)=$ const $\approx 1$ is volume fraction of monomer units in the matrix, and

$$
s(z)=s \cdot\left(\frac{R+z}{R}\right)^{i-1}=\frac{i N_{A} v_{A}^{3}}{R_{i}} \cdot\left(\frac{R+z}{R}\right)^{i-1} \quad i=1,2,3
$$

is the area per $B$ block at distance $z$ from the surface of the $A$-domain.

Substituting of eq 8 into eq 7 and subsequent integration leads to

$$
\frac{F_{B}^{(i)}}{k_{B} T}=\frac{3 \kappa_{B}^{2}}{2 v_{B} l_{B} b_{B}} \int_{0}^{D} z^{2} \phi_{B}(z) s(z) d z
$$

After performing integration in eq 10 using eq 9, one finds

$$
\frac{F_{B}^{(i)}}{k_{B} T}=\frac{D_{i}^{2} v_{A}}{N_{B} v_{B} l_{B} b_{B}} \eta_{B}^{2} \frac{x}{\left[(1+x)^{1 / i}-1\right]^{2}} g_{i}(x)=\frac{R_{i}^{2}}{N_{A} l_{A} b_{A}} \eta_{B}^{2} g_{i}(x) \beta
$$

with ratios

$$
\begin{gathered}
\beta=\frac{v_{B} l_{A} b_{A}}{v_{A} l_{B} b_{B}}, \\
x=\frac{v_{B} N_{B}}{v_{A} N_{A}}=\frac{V_{B}}{V_{A}},
\end{gathered}
$$

and morphology-dependent functions

$$
g_{i}(x)=\frac{\pi^{2}}{8} \begin{cases}x, & i=1 \\ 6 x^{-2}\left[(1+x)^{1 / 2}-1\right]^{4}\left[\frac{1}{4}+\frac{1}{3}\left[(1+x)^{1 / 2}-1\right]^{-1}\right], & i=2 \\ 9 x^{-2}\left[(1+x)^{1 / 3}-1\right]^{5}\left[\frac{1}{5}+\frac{1}{2}\left[(1+x)^{1 / 3}-1\right]^{-1}+\frac{1}{3}\left[(1+x)^{1 / 3}-1\right]^{-2}\right] & i=3\end{cases}
$$

\subsection{Free energy per molecule $(i=1,2,3)$}

The free energy per block copolymer molecule can be presented as

$$
\frac{F^{(i)}}{k_{B} T}=\frac{F_{B}^{(i)}+F_{A}^{(i)}+F_{s}^{(i)}}{k_{B} T}=
$$




$$
\frac{R_{i}^{2}}{N_{A} l_{A} b_{A}} \eta_{B}^{2} g_{i}(x) \beta+\frac{R_{i}^{2}}{N_{A} l_{A} b_{A}} \eta_{A}^{2} C_{i}+\frac{i \gamma N_{A} v_{A}}{l_{A}^{2} R_{i}} \equiv \frac{R_{i}^{2}}{N_{A} l_{A} b_{A}} \Phi_{i}(x)+\frac{i \gamma N_{A} v_{A}}{l_{A}^{2} R_{i}}
$$

where $\gamma=\widetilde{\gamma} l_{A}^{2} / k_{B} T$ is the surface free energy per area $l_{A}^{2}$ measured in units of the thermal energy $k_{B} T$, and

$$
\Phi_{i}(x)=\eta_{B}^{2} g_{i}(x) \beta+\eta_{A}^{2} \mathrm{~b}_{i}
$$

Minimization of the free energy in eq 15 with respect to $R_{i}$ provides geometrical characteristics of the superstructure with morphology $i=1,2,3$ : the radius of domains

$$
R_{i}=\left(\frac{i \gamma N_{A}^{2} v_{A} b_{A}}{2 \Phi_{i}(x) l_{A}}\right)^{1 / 3}
$$

and the size of Wigner-Seitz cell

$$
D_{i}+R_{i}=R_{i}(1+x)^{1 / i}
$$

The aggregation number $Q$ in the equilibrium spherical aggregate $(i=3)$ is given by

$$
Q=\frac{4 \pi}{3} \frac{R_{3}^{3}}{N_{A} v_{A}}=2 \pi \frac{\gamma N_{A} b_{A}}{\Phi_{3}(x) l_{A}}
$$

The number $h^{-1}$ of macromolecules per unit length of cylinder $(i=2)$ yields

$$
1 / h=\pi \frac{R_{2}^{2}}{N_{A} v_{A}}=\pi\left(\frac{\gamma b_{A}}{\Phi_{2}(x) l_{A}}\right)^{2 / 3}\left(\frac{N_{A}}{v_{A}}\right)^{1 / 3}
$$

while grafting density $s^{-1}$ of macromolecules in the lamellar phase $(i=1)$ reduces to

$$
1 / s=\frac{R_{1}}{N_{A} v_{A}}=v_{A}^{-2 / 3}\left(\frac{\gamma b_{A}}{2 \Phi_{i}(x) l_{A}}\right)^{1 / 3} N_{A}^{-1 / 3}
$$

The free energy per molecule in superstructure of morphology $i$ yields

$$
\frac{F^{(i)}(x)}{k_{B} T}=\frac{3}{2^{2 / 3}}(i \gamma)^{2 / 3} N_{A}^{1 / 3} \Phi_{i}^{1 / 3}(x)\left(\frac{v_{A}^{2 / 3}}{l_{A}^{5 / 3} b_{A}^{1 / 3}}\right)
$$

with all the architectural details of constituent blocks incorporated in $\Phi_{i}$ (eq 16) via topological ratios $\eta_{A}$ and $\eta_{B}$.

Equations 18, 19,20 indicate that grafting density of the chains in each of the superstructures decreases upon branching of one or both of the blocks (i.e., with increasing $\eta_{A}$ and/or $\eta_{B}$ )

\section{Samples characterization}

Here we collect selected experimental data discussed in the main text and figures. 
Table S1. Selected data for $l-b b$ copolymer $^{6}$ with $n_{B} \ll M_{B}$. In the sample lable $M_{x: y}: N_{z}, x$ - number of repeat units in $b b$ backbone, $y$ - mollar mass of PS side chain, $z$ - number of repeat units in linear block. $f_{B}=$ $V_{B} /\left(V_{B}+V_{A}\right)$ - volume fraction of $b b$ block PS, $n_{B}$ - degree of polymerization of PS side chains (estimated as ratio of molar masses of PS side chain ${ }^{6}$ and $M_{0}=104 \mathrm{~g} / \mathrm{mol}$ of PS monomer unit, rounded up to interger number), $\eta_{B}=$ $0.8 n_{B}^{1 / 4}$ is topological ratio for PS block with the numerical coefficient $a_{B}=$ $0.65,2 R_{i}$ - size of domain formed by $l$ block $A$, evaluated from experimentally measured spacing $d_{i}$ between centers of neighboring domains according to eq 2 as $2 R_{i}=d_{i}\left(1-f_{B}\right)^{1 / i}$ for $i=1$ (lamellae, L), $i=2$ (cylinders, C) and $i=3$ (spheres, $\mathrm{S})$.

\begin{tabular}{|l|l|l|l|l|l|l|l|}
\hline$N$ & \multicolumn{1}{|c|}{ Sample } & $f_{B}$ & $n_{B}$ & $\beta^{1 / 2} \eta_{B}$ & & $d_{i}(\mathrm{~nm})$ & $2 R_{i}(\mathrm{~nm})$ \\
\hline 1 & $M_{100: 1590}: N_{1000}$ & 0.33 & 15 & 1.57 & $\mathrm{~L}$ & $70 \pm 4$ & $47 \pm 3$ \\
\hline 2 & $M_{150: 687}: N_{1500}$ & 0.19 & 7 & 1.30 & $\mathrm{~L}$ & $85 \pm 4$ & $69 \pm 3$ \\
\hline 3 & $M_{200: 1850}: N_{2000}$ & 0.37 & 18 & 1.65 & $\mathrm{~L}$ & $98 \pm 10$ & $62 \pm 6$ \\
\hline 4 & $M_{250: 2120}: N_{2000}$ & 0.45 & 20 & 1.69 & $\mathrm{~L}$ & $116 \pm 17$ & $64 \pm 9$ \\
\hline 5 & $M_{100: 3730}: N_{400}$ & 0.69 & 36 & 1.96 & $\mathrm{C}$ & $72 \pm 4$ & $40 \pm 2$ \\
\hline 6 & $M_{100: 3740}: N_{1000}$ & 0.54 & 35 & 1.95 & $\mathrm{C}$ & $87 \pm 12$ & $59 \pm 8$ \\
\hline 7 & $M_{100: 4560}: N_{1000}$ & 0.59 & 43 & 2.05 & $\mathrm{C}$ & $112 \pm 11$ & $72 \pm 7$ \\
\hline 8 & $M_{250: 3110}: N_{1000}$ & 0.68 & 30 & 1.87 & $\mathrm{C}$ & $93 \pm 3$ & $53 \pm 2$ \\
\hline 9 & $M_{150: 3750}: N_{1500}$ & 0.54 & 36 & 1.96 & $\mathrm{C}$ & $109 \pm 9$ & $59 \pm 6$ \\
\hline 10 & $M_{150: 5700}: N_{1500}$ & 0.64 & 55 & 2.18 & $\mathrm{C}$ & $111 \pm 17$ & $67 \pm 10$ \\
\hline 11 & $M_{150: 6750}: N_{2000}$ & 0.62 & 65 & 2.27 & $\mathrm{C}$ & $138 \pm 15$ & $85 \pm 9$ \\
\hline 12 & $M_{150: 4630}: N_{2000}$ & 0.53 & 45 & 2.07 & $\mathrm{C}$ & $118 \pm 11$ & $78 \pm 8$ \\
\hline 13 & $M_{150: 4540}: N_{2000}$ & 0.52 & 44 & 2.06 & $\mathrm{C}$ & $77 \pm 3$ & $53 \pm 2$ \\
\hline 14 & $M_{200: 3240}: N_{2000}$ & 0.50 & 31 & 1.89 & $\mathrm{C}$ & $114 \pm 6$ & $81 \pm 4$ \\
\hline 15 & $M_{200: 3620}: N_{2000}$ & 0.53 & 35 & 1.95 & $\mathrm{C}$ & $126 \pm 13$ & $86 \pm 9$ \\
\hline 16 & $M_{150: 5790}: N_{1500}$ & 0.64 & 56 & 2.19 & $\mathrm{~S}$ & $123 \pm 16$ & $88 \pm 11$ \\
\hline 17 & $M_{250: 3910}: N_{1000}$ & 0.73 & 38 & 1.99 & $\mathrm{~S}$ & - & - \\
\hline 18 & $M_{200: 6410}: N_{2000}$ & 0.67 & 62 & 2.24 & $\mathrm{~S}$ & - & - \\
\hline 19 & $M_{250: 5270}: N_{2000}$ & 0.67 & 51 & 2.18 & $\mathrm{~S}$ & - & - \\
\hline 20 & $M_{250: 6170}: N_{2000}$ & 0.70 & 59 & 2.22 & $\mathrm{~S}$ & - & - \\
\hline 21 & $M_{250: 6280}: N_{2000}$ & 0.70 & 60 & 2.23 & $\mathrm{~S}$ & - & - \\
\hline 22 & $M_{350: 4160}: N_{2000}$ & 0.68 & 40 & 2.01 & $\mathrm{~S}$ & - & - \\
\hline 23 & $M_{350: 5980}: N_{2000}$ & 0.75 & 57 & 2.20 & $\mathrm{~S}$ & - & - \\
\hline 24 & $M_{350: 7020}: N_{2000}$ & 0.78 & 68 & 2.30 & $\mathrm{~S}$ & - & - \\
\hline
\end{tabular}


Table S2. Selected data for PS-PEO $b b-b b$ diblock copolymer ${ }^{5}$ with PEO block as block $B . M_{A}$ and $M_{B}$ are numbers of repeat units in the backbones of PS and PEO blocks, respectively. $r_{0, A}$ and $r_{0, B}$ are Gaussian sizes of the side chains in Angstrems. Although in last two samples $n_{A}<M_{A}$, we keep them as a decrease in $M_{A}$ promotes formation of cylindrical (C) phase compared to samples with $M_{A}>n_{A}$.

\begin{tabular}{|l|l|l|l|l|l|l|l|l|}
\hline Sample & & $f_{B}$ & $M_{A}$ & $M_{B}$ & $n_{A}$ & $r_{0, A}(\AA)$ & $n_{B}$ & $r_{0, B}(\AA)$ \\
\hline $2 \mathrm{k}-45$ & $\mathrm{~L}$ & 0.45 & 49 & 60 & 34 & 39 & 45 & 43 \\
\hline $2 \mathrm{k}-71$ & $\mathrm{~L}$ & 0.71 & 21 & 92 & 34 & 39 & 45 & 43 \\
\hline $2 \mathrm{k}-79$ & $\mathrm{C}$ & 0.79 & 14 & 89 & 34 & 39 & 45 & 43 \\
\hline
\end{tabular}

Table S3. Selected data for PS-PLA $b b-b b$ diblock copolymer ${ }^{1,4}$ with PLA block as block $B$. Size of PS domain in lamella morphology (L) is estimated as $2 R_{1}=d_{1}\left(1-f_{B}\right)$. Molecular mass of $b b-b b$ sample $X$ with $\mathrm{C}$ ' morphology and diameter of inverted (PLA) cylinder $47 \mathrm{~nm}$, was estimated from GPC as $187.3 \mathrm{~kg} / \mathrm{mol} .{ }^{4}$ Total number of monomer units $N_{A}$ in PS block was estimated as $M_{P S} / M_{0}{ }^{4}$

\begin{tabular}{|l|l|l|l|l|l|l|l|l|l|l|}
\hline Sample & $f_{B}$ & & $n_{A}$ & $r_{0, A}(\AA)$ & $n_{B}$ & $r_{0, B}(\AA)$ & $\left(\frac{r_{0, B}}{r_{0, A}}\right)^{1 / 2}$ & $d_{i}$ & $2 R_{i}(n m)$ & $N_{A}$ \\
\hline SL-1 & 0.37 & $\mathrm{~L}$ & 31 & 37.3 & 36 & 36.7 & 0.99 & 153 & 96 & 13020 \\
\hline SL-2 & 0.29 & $\mathrm{~L}$ & 31 & 37.3 & 36 & 36.7 & 0.99 & 163 & 116 & 13020 \\
\hline SL-3 & 0.57 & $\mathrm{~L}$ & 29 & 36.1 & 40 & 38.7 & 1.04 & 105 & 45 & 6090 \\
\hline SL-4 & 0.40 & $\mathrm{~L}$ & 29 & 36.1 & 19 & 26.7 & 0.86 & 100 & 60 & 6090 \\
\hline SL-5 & 0.43 & $\mathrm{~L}$ & 55 & 49.7 & 50 & 43.3 & 0.93 & 126 & 72 & 11550 \\
\hline $\mathrm{X}^{4}$ & 0.30 & $\mathrm{C} /$ & 46 & 45.5 & 14 & 22.9 & 0.71 & & 47 & 1260 \\
\hline
\end{tabular}


Table S4. Selected data from $\operatorname{ref}^{7}$ for $b b-b b$ polymer PS-PDMS with PS block as block $B$.

\begin{tabular}{|l|l|l|l|l|l|l|l|}
\hline Sample & $f_{B}$ & & $n_{B}$ & $r_{0, B}(\AA)$ & $n_{A}$ & $r_{0, A}(\AA)$ & $\left(\frac{r_{0, B}}{r_{0, A}}\right)^{1 / 2}$ \\
\hline $3.1 \mathrm{k}-\mathrm{L}-50 \%$ & 0.48 & $\mathrm{~L}$ & 29 & 37 & 65 & 47 & 0.89 \\
\hline $3.1 \mathrm{k}-\mathrm{L}-40 \%$ & 0.38 & $\mathrm{C} \prime$ & 29 & 37 & 65 & 47 & 0.89 \\
\hline $4.7 \mathrm{k}-\mathrm{L}-50 \%$ & 0.48 & $\mathrm{~L}$ & 45 & 45 & 65 & 47 & 0.98 \\
\hline $4.7 \mathrm{k}-\mathrm{L}-40 \%$ & 0.38 & $\mathrm{~L}$ & 45 & 45 & 65 & 47 & 0.98 \\
\hline
\end{tabular}

Table S5. Selected data for PMMA-PDMS-PMMA $l-b b-l$ block copolymer $^{8}$ treated as 2 diblocks PMMA-PDMS/2. Degree of polymerization of side chain in $b b$ block PDMS (block $B) n_{B}=14$ in all samples, degree of polymerization $N_{A}$ of $l$ PMMA block (block $A$ ) is varied. Experimental diameter of PMMA spherical domains is $2 R_{3}, f_{B}$ is volume fraction of PDMS in triblock copolymer. The value of $\beta^{1 / 2}\left(\eta_{B} / \eta_{A}\right) \approx 1.25$ was calculated from the first of eqs 31 with $\alpha_{B}=0.50$ and molecular parameters of PMMA and PDMS blocks listed in Table 1.

\begin{tabular}{|l|l|l|l|l|}
\hline Sample & & $N_{A}$ & $f_{B}$ & $2 R_{3}(\mathrm{~nm})$ \\
\hline 300_1 & $\mathrm{S}$ & 100 & 0.95 & 20.8 \\
\hline 300_2 & $\mathrm{S}$ & 166 & 0.92 & 25.5 \\
\hline $300 \_3$ & $\mathrm{~S}$ & 276 & 0.87 & 30.2 \\
\hline $900 \_1$ & $\mathrm{~S}$ & 190 & 0.97 & 24.3 \\
\hline $900 \_2$ & $\mathrm{~S}$ & 325 & 0.95 & 32.3 \\
\hline $900 \_3$ & $\mathrm{~S}$ & 656 & 0.90 & 50.9 \\
\hline $900 \_4$ & $\mathrm{~S}$ & 1235 & 0.83 & \\
\hline 1200_1 & $\mathrm{S}$ & 360 & 0.95 & 33.4 \\
\hline 1200_2 & $\mathrm{S}$ & 480 & 0.83 & 42.8 \\
\hline 1200_3 & $\mathrm{S}$ & 810 & 0.89 & 45.7 \\
\hline 1500_3 & $\mathrm{S}$ & 867 & 0.91 & 50.3 \\
\hline 1800_3 & $\mathrm{S}$ & 780 & 0.93 & \\
\hline
\end{tabular}


Table S6. Selected data for PS-PDMS-PS $l-b b-l$ block copolymer. ${ }^{9} f_{B}$ - volume fraction of PDMS in triblock copolymer. Degree of polymerization $N_{A}$ of PS block was estimated as $N_{A}=M_{n}\left(1-f_{B}\right) /\left(2 M_{0}\right)$ with $M_{0}=104$ $\mathrm{g} / \mathrm{mol}$. Superscript in sample lable indicates molar mass of side chain in $K D a$. Number $n_{B}$ of PS monomers in the side chain was estimated as ratio of its molar mass and $M_{0}$, and rounded up to interger value.

\begin{tabular}{|l|l|l|l|l|l|l|}
\hline & $M_{n}(\mathrm{KDa})$ & $N_{A}$ & $n_{B}$ & $\beta^{1 / 2} \frac{\eta_{B}}{\eta_{A}}$ & $f_{B}$ & $2 R_{3}(\mathrm{~nm})$ \\
\hline $\mathrm{S}_{500}^{1}$ & 510 & 147 & 10 & 1.06 & 0.94 & $23.8 \pm 3.4$ \\
\hline $\mathrm{S}_{500}^{5}$ & 550 & 158 & 48 & 1.56 & 0.94 & $15.8 \pm 2.6$ \\
\hline $\mathrm{S}_{1000}^{0.8}$ & 990 & 95 & 8 & 1.01 & 0.98 & $42.4 \pm 8.4$ \\
\hline $\mathrm{S}_{1000}^{1}$ & 1070 & 103 & 10 & 1.06 & 0.98 & $30.4 \pm 6.4$ \\
\hline $\mathrm{S}_{1000}^{5}$ & 922 & 89 & 48 & 1.56 & 0.98 & $7.6 \pm 0.6$ \\
\hline
\end{tabular}

Table S7. Molecular parameters of copolymer blocks

\begin{tabular}{|l|l|l|l|l|l|l|}
\hline & aPP & PS & PEO & PMMA & PDMS & PLA \\
\hline$M_{0}(\mathrm{~g} / \mathrm{mol})$ & 43 & 104 & 44 & 100 & 74 & 72 \\
\hline$l(\AA)$ & 2.5 & 2.5 & 3.75 & 2.5 & 2.5 & 3.75 \\
\hline$b(\AA)$ & 11 & 18 & 11 & 17 & 13 & 10 \\
\hline$\rho\left(\mathrm{g} / \mathrm{cm}^{3}\right)$ & 0.79 & 0.97 & 1.06 & 1.18 & 0.90 & 1.25 \\
\hline$v\left(\AA^{3}\right)$ & 90 & 178 & 69 & 141 & 136 & 96 \\
\hline$(l b)^{3 / 4} / v^{1 / 2}$ & 1.26 & 1.30 & 1.44 & 1.40 & 1.18 & 1.54 \\
\hline
\end{tabular}

Table S8. Ratios $\beta=\left(l_{A} b_{A} v_{B}\right) /\left(l_{B} b_{B} v_{A}\right)$ for selected $A B$ block copolymers

\begin{tabular}{|l|l|l|l|l|}
\hline$A-B$ & PS-PEO & PLA-PS & PMMA-PDMS & aPP-PS \\
\hline$\beta$ & 0.42 & 1.54 & 1.26 & 1.21 \\
\hline
\end{tabular}




\section{References}

[1] J. Rzayev Synthethis of polystyrene-polylactide bottlebrush block copolymers and their melt self-assembly into large domain nanostructures. Macromolecules 2009, 42, 2135-2141

[2] Semenov, A. N. Contribution to the Theory of Microphase Layering in Block-Copolymer Melts. Sov. Phys. JETP 1985, 61, 733-742.

[3] Lebedeva, I.O.; Zhulina, E. B. ; Leermakers, F. A. M.; Borisov O.V. Dendron and Hyperbranched Polymer Brushes in Good and Poor Solvents. Langmuir 2017, 33, 1315-1325

[4] J. Bolton, T.S. Baile, J. Rzayev. Large pore size nanoporous materials from self-assembly of asymmetric bottlebrush block copolymers. Nano Lett. 2011, 11, 998-1001

[5] Y. Gai, D.-P. Song, B. M. Yavitt, J. J. Watkins. Polystyrene-blockpoly(ethylene oxide) Bottlebrush Block CopolymerMorphology Transitions: Influence of Side Chain Length and Volume Fraction. Macromolecules 2017, 50, 1503-1511

[6] M.B. Runge, C.E. Lipscomb, L.R. Ditzler, M.K. Mahanthappa, A.V. Tivanski, N.B. Bowden. Investigation of the Assembly of Comb Block Copolymers in the Solid State. Macromolecules 2008, 41, 7687-7694

[7] H.-F. Fei, B. M. Yavitt, X. Hu, G. Kopanati, A. Ribbe, J. J. Watkins. Influence of Molecular Architecture and Chain Flexibility on the Phase Map of Polystyrene-block-poly(dimethylsiloxane) Brush Block Copolymers. Macromolecules 2019, 52, 6449-6457

[8] C. Clair, A. Lallam, M. Rosenthal, M. Sztucki, M.VatankhahVarnosfaderani, A.N. Keith, Y. Cong, H. Liang, A.V. Dobrynin, S.S. Sheiko, D.A. Ivanov. ACS Macro Lett. 2019, 8, 530-534

[9] S. Nian, H. Lian, Z. Gong, M. Zhernenkov, J. Qin, L.-H. Cai. Molecular Architecture Directs Linear-Bottlebrush-Linear Triblock Copolymers to Self-Assemble to Soft Reprocessable Elastomers. ACS Macro Lett. 2019, $8,1528-1534$ 